\title{
New Generation Vaccines (4th Edition)
}

\section{Francesco M Marincola}

Infectious Disease \& Immunogenetics Section, Department of Transfusion Medicine, Clinical Center \& Trans-NIH Center for Human Immunology, National Institutes of Health, Bethesda, ML 20892, USA

- Tel.: +1 3014514967 . Fax: +1 3014021360 .fmarincola@mail.cc.nih.gov

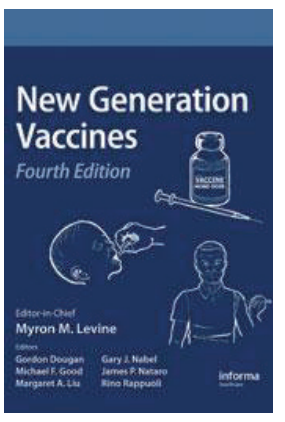

Edited by Levine MM

Informa Healthcare,

NY, USA

ISBN 978-1-420060-

73-7, 1-420060-73-2

(2010)
During the last decade, several successes have been achieved in the field of vaccinology that maintain its paramount position as the leading hope for the treatment of most acute and chronic diseases worldwide. However, challenges remain at both the logistic and the scientific level. The fourth edition of New Generation Vaccines updates the reader concerning recent scientific and clinical advances in vaccinology, clearly framing the discussion in the context of future challenges and the corresponding strategies regarding how to pursue the solutions.
This decade has clearly demonstrated that vaccination strategies aimed at the induction of antigen-specific immune responses cannot stand alone as tools to achieve protection against an infectious agent, even when vaccines are successful in achieving their biological goal of inducing adaptive recognition. This observation raises the question of how successful vaccines, such as the ones against influenza, smallpox and polio virus (to stick to viral examples), might work while other ones, such as those against hepatitis $\mathrm{C}$ virus or HIV, have clearly failed. It could be hypothesized that successful vaccines may face a pathogen characterized by more stable antigenic properties and are thus less likely to escape immune recognition; alternatively, it could be argued that the biological properties of those viruses against which vaccines successfully compete may be different, particularly in the way they affect the environment in which they reside. It could be hypothesized that vaccine-sensitive viruses induce the activation of powerful proinflammatory stimuli around the infected cells that may be critical for the activation of antigen-specific $T$ cells induced by the vaccine at a distant site. Without that secondary activation, antigen-specific $\mathrm{T}$ cells cannot effectively carry out their effector functions. This may not be critical in conditions in which viruses are predominantly lytic and can be neutralized in the circulation by antibodies in addition to the fact that they cannot linger in infected cells for too long since infection leads to the demise of the infected cells. However, for nonlytic viruses, persistence of infection becomes a problem if effector $T$ cells cannot completely clear the infected cells, as it most often appears to be the case when vaccines do not work. Interestingly, this problem is not unique to vaccines against chronic viral or bacterial infections, but also appears to apply to other pathologies in which a cognate immune response has been documented, such as in cancer. Anticancer vaccines have been very successful in inducing antigen-specific immune responses, but these are not associated with the clearance of antigen-bearing cells. This paradoxical observation clearly exemplifies the limitations of inducing antigen-specific immune responses from the afferent arm of the vaccine that cannot be further activated and maintained at the efferent arm by a conducive proinflammatory milieu. Perhaps the biggest difference between successful and unsuccessful vaccine models resides in the intrinsic biology of the pathogen itself, which can induce potent inflammation at the affected site in the event of success but minimally affects the microenvironment in other cases. The early chapters of this textbook address in detail the basic understanding of the biology modulating the effectiveness of vaccines and, most importantly, the recent progress in the understanding of the 
"...this text book spans topics that cover adjuvant preparation, vaccine formulation, route of administration and delivery in great detail." relevance of innate (nonantigen-specific) immune responses in playing an adjuvant role, not only at the vaccine site, but also in the affected organ. In my view, this is one of the most compelling additions in the fourth edition.

Discussions regarding the logistics and the economics of vaccine delivery, particularly in developing countries, are also described in detail and perhaps represents the hopeful facet of the problem, since so much could be done with what is already available to improve human health in populations that are at risk across the world. Reading through the pages, it is striking how much good could be derived from a little effort, and yet how insurmountable some challenges can be because of peripheral problems that can only be understood by those who are working on the front line. There are several compelling chapters covering this topic, including the exciting description of the development of the Global Alliance for Vaccines and Immunization (GAVI).

It is impossible to give details about all the scientific topics covered by this compendium; this textbook spans topics that cover adjuvant preparation, vaccine formulation, route of administration and delivery in great detail. Specifics regarding various bacterial and viral diseases are covered exhaustively, including old and new challenges. In particular, these include novel challenges and the progress made in confronting them: the identification of effective strategies against the threat of bioterrorism and the rapid progress in developing reagents against emergent infectious diseases, such as SARS, and more generally, the human pandemic of influenza. Finally, overviews are presented concerning nonconventional vaccine approaches against cancer, neurodegenerative disorders and autoimmune and chronic inflammatory diseases. As with any attempt to cover a broad field of investigation, this textbook may have left some topics unsatisfactorily discussed. However, to quote the editors themselves: "...even with 89 chapters, it is impossible to include in a single text of research vaccinology updates on vaccines against every disease target and all new technologies. ... The editors have tried to minimize these voids," and I think that they did a great job. 